\title{
A ECOLOGIA DE ESTRADA SOB A ÓTICA DO LICENCIAMENTO AMBIENTAL
}

\author{
Gustavo Aveiro Lins \\ Mestre em Engenharia Ambiental \\ Universidade federal do Rio de Janeiro (UFRJ) \\ $\bowtie$ gu.lins@terra.com.br \\ Luiz Gustavo Escorcio Bezerra \\ Mestre em Direito Ambiental \\ University College London (UCL) \\ $\triangle$ luizgustavo.bezerra@gmail.com \\ Maurício Jorge Pereira da Mota \\ Doutor em Direito Civil \\ Universidade do estado do Rio de Janeiro (UERJ) \\ $\triangle$ mjmota1@gmail.com \\ Oscar Rocha-Barbosa \\ Pós-Doutorado em Zoologia - Universitat de Barcelona \\ \or-barbosa@hotmail.com \\ Josimar Ribeiro de Almeida \\ Doutor - Professor Universidade do Estado do Rio de Janeiro (UERJ) \\ Pesquisador Senior Centro de Engenharia Nuclear/USP - RJ - Brasil \\ $\bowtie$ jralmeida@usp.br
}

\section{Resumo:}

As rodovias são fundamentais para a manutenção e crescimento da economia de um país, lamentavelmente, vários estudos tem mostrado a relação das rodovias com importantes impactos ambientais, diante disso entende-se a grande importância do aprofundamento do conhecimento da questão da gestão ambiental em rodovias, sobretudo a questão legal. A conclusão do artigo é que o licenciamento ambiental é um processo importante no gerenciamento ambiental das rodovias, no entanto falta seriedade no monitoramento pós construção, condição necessária para que se avalie a efetividade das medidas mitigadoras adotadas.

Palavras-chave: Licenciamento; Rodovias; Ecologia de rodovia.

\section{THE ROAD OF ECOLOGY FROM THE PERSPECTIVE OF ENVIRONMENTAL LICENSING}

\begin{abstract}
:
The highways are essential for the maintenance and growth of the economy of a country, unfortunately, several studies have shown a list of the highways with significant environmental impacts, before it means the great importance of the deepening of the
\end{abstract}


environmental management issue of knowledge in highways, especially the legal question. The conclusion of the article is that the environmental licensing is an important process in environmental management of highways, however lack seriousness in the post construction monitoring, as required for an evaluation of the effectiveness of mitigation measures taken.

Keywords: Licensing; Highways; Highway ecology.

\section{EL CAMINO DE LA ECOLOGÍA DESDE LA PERSPECTIVA DE LICENCIA AMBIENTAL}

\section{Resumen:}

Las carreteras son esenciales para el mantenimiento y crecimiento de la economía de un país, por desgracia, varios estudios han demostrado una lista de las carreteras con impactos ambientales significativos, antes de que significa la gran importancia de la profundización del tema de gestión ambiental de los conocimientos en las carreteras, especialmente la questçao legal. La conclusión del artículo es que la licencia ambiental es un proceso importante en gestión ambiental de autopistas, sin embargo carece de seriedad en el seguimiento posterior a la construcción, como se requiere para una evaluación de la eficacia de las medidas de mitigación adoptadas.

Palabras clave: Concesión de licencias; Carreteras; Ecología carretera.

\section{ESTADO DA ARTE}

O sistema rodoviário exerce um papel fundamental no desenvolvimento da sociedade. Rodovias melhoram o acesso às áreas produtoras e turísticas, incentivando a distribuição de renda e o desenvolvimento social (PERZ et al., 2007). No entanto, rodovias também causam impactos ambientais negativos (FORMAN e ALEXANDER, 2007). Estes impactos negativos envolvem fragmentação de habitat (FORMAN e DEBLINGER, 2000; SOARES-FILHO et al., 2004, KOCIOLEK et al., 2011), alterações na hidrologia (JONES et al., 2000), poluição do solo (REIJNEN et al., 1995), introdução de espécies exóticas (FORMAN et al., 2003), facilitação da atividade de caça (FORMAN e ALEXANDER, 2007) além do atropelamento de animais (BAGER, 2003). A mortalidade de animais nas rodovias tem sido apontada em muitos casos, como um elemento de grande contribuição para a redução de biodiversidade de várias espécies de vertebrados (COFFIN, 2007; TROMBULAK e FRISSELL, 2000). Dos impactos de rodovias à biodiversidade, o atropelamento de fauna silvestre é um dos mais visíveis e certamente um dos mais importantes (LODÉ, 2000, CLEVENGER et. al., 2003, TAYLOR e GOLDINGAY, 2004). 
A preocupação com os impactos das estradas na fauna resultou nos esforços de mitigar estes efeitos (FORMAN et al., 2003). Uma das formas de mitigar estes impactos é proporcionar a travessia da fauna local com segurança, isso pode ser feito a partir da implantação de passagens de fauna (BECKMANN et al., 2010). As passagens de fauna, ou zoopassagens têm como princípio restabelecer a conectividade entre paisagens (ABRA, 2012), evitando atropelamentos e permitindo o fluxo genético entre populações selvagens e assim garantir a viabilidade populacional das espécies prejudicadas pela fragmentação (CORLATTI et al., 2009). Essas passagens podem ser caracterizadas como túneis subterrâneos, pontes verdes, aéreas ou mesmo como as próprias drenagens de escoamento da água superficial, instaladas sob as rodovias. Todavia, o uso das passagens pelos animais pode ser influenciado por diversos fatores: conformação da estrutura de engenharia, comprimento, localização e características da região do entorno (CORLATTI et al., 2009).

A importância de estudos relacionados aos impactos ambientais das estradas em ecossistemas brasileiros, é de grande importância tendo em vista que o transporte rodoviário no Brasil é o principal sistema logístico contando com uma rede de 1.751 .868 quilômetros de rodovias por onde passam $56 \%$ de todas as cargas movimentadas no território brasileiro. Estudos sobre atropelamento de animais silvestres citam que, após a construção de uma estrada, a fauna de uma determinada região pode ser severamente prejudicada, inclusive com a extinção local. No território brasileiro é no estado de Minas Gerais que o problema mostrase ainda mais grave pelo seu sistema rodoviário ser o maior do Brasil (SILVA et al, 2011). Diante disso, é urgente que métodos já utilizados para mitigar tais impactos (zoopassagens, por exemplo) sejam avaliados sob diferentes situações, e que o conhecimento gerado seja utilizado no planejamento e manutenção da infra-estrutura de transporte. Apesar deste problema, a ecologia de estradas ainda é um tema pouco estudado no Brasil, segundo Lauxen (2012), na revisão bibliográfica feita pelo autor, dos 230 artigos específicos sobre ecologia de estradas, apenas 14 eram de trabalhos nacionais.

O grande impacto negativo da instalação de rodovias para a fauna e a biodiversidade geralmente se contrapõe aos predominantemente positivos impactos socioeconômicos. É justamente no licenciamento ambiental que são analisados os impactos negativos e positivos de uma determina atividade e exigidas, caso o empreendimento proposto seja considerado viável, uma série de medidas de mitigação e compensação dos impactos. As licenças ambientais são então emitidas sendo impostas diversas condicionantes, verdadeiras 
obrigações a serem cumpridas pelo empreendedor a validarem a regularidade do empreendimento licenciado.

O licenciamento ambiental é um dos principais instrumentos da Lei N. ${ }^{\circ} 6.938 / 81$, que estabelece as diretrizes da Política Nacional de Meio Ambiente. Trata-se de um procedimento administrativo que visa ao controle das obras e ou atividades que possam resultar intervenções ao meio ambiente, definindo direitos e obrigações para o exercício das atividades licenciadas. Encontra guarida constitucional no artigo 225 , em seu $\S 1^{\circ}$, inciso IV, e no artigo 10 da Lei da Política Nacional de Meio Ambiente.

O Art. 19 do Decreto 99.274/90 enuncia as licenças ambientais que serão expedidas pelo Poder Público, quais sejam: (i) Licença Prévia (LP), na fase preliminar do planejamento de atividade, contendo requisitos básicos a serem atendidos nas fases de localização, instalação e operação, observados os planos municipais, estaduais ou federais de uso do solo; (ii) Licença de Instalação (LI), autorizando o início da implantação, de acordo com as especificações constantes do Projeto Executivo aprovado; e (iii) Licença de Operação (LO), autorizando, após as verificações necessárias, o início da atividade licenciada e o funcionamento de seus equipamentos de controle de poluição, de acordo com o previsto nas Licenças Prévia e de Instalação.

Além destas licenças, o Conselho Nacional de Meio Ambiente - CONAMA ainda pode definir licenças ambientais específicas de acordo com a natureza, características e peculiaridades de determinada atividade, e a respectiva compatibilização com as etapas de implantação e operação.

No âmbito do CONAMA, algumas resoluções devem ser destacadas no que tange ao licenciamento ambiental. A resolução CONAMA 01/86 determina que dependerá de elaboração de estudo de impacto ambiental (EIA) e respectivo relatório de impacto ambiental (RIMA), a serem submetidos à aprovação do órgão ambiental competente, as atividades modificadoras do meio ambiente, em rol exemplificativo de empreendimentos trazido pela referida resolução. Estabelece, por exemplo, que a construção de estradas de rodagem com duas ou mais faixas de rolamento devem ser precedidas da elaboração EIA/RIMA. Fundamental ainda é a Resolução 237/97 que regulamenta diversos aspectos de licenciamento ambiental estabelecidos na Política Nacional do Meio Ambiente. 
Desta forma, é o licenciamento ambiental o instrumento jurídico por excelência, no qual o órgão ambiental competente pode definir o traçado menos impactante, discutindo as alternativas locacionais do empreendimento, e exigir as medidas adequadas para mitigação dos impactos ambientais da instalação de estradas. Neste ponto, concordamos com Lauxen (2012) de que "a maior oportunidade de se minimizar os impactos se apresenta quando da implantação de uma nova rodovia, pois por ocasião da definição de seu traçado podem ser evitadas as áreas ecologicamente mais sensíveis, em termos de estado de conservação e extensão da cobertura vegetal" (LAUXEN, 2012, pg.8).

É nesta fase inicial do licenciamento que é elaborado o EIA/RIMA, em linha com os projetos básicos de engenharia das rodovias, que indicam a necessidade de inserção, a quantidade, a tipologia e a localização sugerida para os dispositivos de travessia de fauna, por exemplo. (BONET e CUNHA, 2012).

Neste mister o EIA/RIMA, espécie de avaliação de impactos ambientais, mostra-se como o mais detalhado e profundo estudo, que pode ser exigido para a instalação de uma nova estrada. São comuns críticas à qualidade destes estudos que, por vezes correm o risco de serem um exaustivo levantamento de informações sem a devida reflexão sobre a verdadeira utilidade das informações para a implantação do empreendimento.

GONÇALVES (2012, p. 217) corrobora este entendimento ao entender que "muitos dos EIAs apenas resultam em uma lista de espécies encontradas no entorno do empreendimento, mas, isso, efetivamente não responde o que deve ser perguntado: 'Quais os impactos do empreendimento?' 'É possível mitigá-los?' 'Como?'”. Esta é uma lacuna que se vencida tem o potencial de se tornar um necessário e forte elo entre o universo acadêmico e os órgãos licenciadores, gerando benefícios para toda sociedade.

Lauxen entende que o licenciamento ambiental pode ter ainda mais protagonismo, ao dizer que "oferece uma excelente oportunidade tanto para discussão de ideias, conceitos $e$ propostas, quanto para geração de conhecimento, em particular se conduzido no aspecto técnico sob critérios cientificamente válidos. Embora sem perder de vista os limites e objetivos para os quais foi implantado, é compatível com o interesse público que as informações produzidas em seu contexto subsidiem a construção de conhecimento aplicável por toda a sociedade, além de ter suas decisões embasadas em procedimentos científicos válidos" (LAUXEN, 2012, p. 67). 


\section{A ECOLOGIA DE ESTRADA SOB A ÓTICA \\ DO LICENCIAMENTO AMBIENTAL}

No cenário brasileiro, o Programa de Aceleração do Crescimento (PAC), com a expansão de infraestruturas e o investimento de 24 bilhões de dólares na construção e manutenção de rodovias entre 2011 e 2014, relaciona-se com o Programa de Rodovias Federais Ambientalmente Sustentáveis (PROFAS), que objetiva regularizar ambientalmente nada menos que $55.000 \mathrm{~km}$ de rodovias nos próximos 20 anos e mostra que o país pretende alinhar o desenvolvimento à sustentabilidade e ao controle/mitigação dos impactos.

\section{CONSIDERAÇÕES FINAIS}

Conclui-se, assim, que o licenciamento ambiental tem papel fundamental no objeto de pesquisa, principalmente porque não existem critérios suficientemente claros para indicação de pontos críticos "e a avaliação da efetividade das medidas mitigadoras adotadas fica restrita ao âmbito dos respectivos processos e técnicos envolvidos". Em contrapartida, o principal motivo da insuficiência de qualidade de dados trazidos pelos EIAs "está vinculado à falta de formulação das perguntas corretas e à não adequação de métodos que possam responder as perguntas em relação ao impacto do empreendimento". Exemplo disso é que apesar de muitos EIAs incluírem a questão do monitoramento de atropelados na parte do Plano Básico Ambiental como um Programa de Monitoramento, falham na ausência de medidas de monitoramento pós construção, que é condição necessária para que se avalie a efetividade e eficácia das medidas adotadas e se avance, enfim, no tratamento dos impactos das rodovias sobre a biodiversidade.

\section{REFERÊNCIAS BIBLIOGRÁFICAS}

ABRA, F. D. Monitoramento e avaliação das passagens inferiores de fauna presentes na rodovia SP-225 no município de Brotas, São Paulo. Dissertação (mestrado). 256 p. Universidade de São Paulo, USP, 2012.

BAGER, A. Repensando as medidas mitigadoras impostas aos empreendimentos rodoviários associados a Unidades de Conservação - Um estudo de caso, In: Bager A (ed) Áreas Protegidas: Conservação no Âmbito do Cone Sul, Bager A, Pelotas, Brasil, pp 159-172, 2003.

BECKMANN, J.O; CLEVENGER, A.P.; HUIJSER, M.P. \& HILTY, J.A. Safe passages - Highways, wildlife, and habitat connectivity.Island Press, 396 p. 2010.

BONET, B.; CUNHA, H.. Medidas Preventivas aos Atropelamentos de Fauna em Rodovias. Monitoramento e mitigação de atropelamentos de fauna. Coordenação Geral de Meio Ambiente. Diretoria de Planejamento e Pesquisa. Coleção Estrada Verde. Brasília, 2012.

BRASIL. Lei Federal no 6938/81. Política nacional do meio ambiente - PNMA. 1981. Disponível em: <http://www.planalto.gov.br/ccivil_03/Leis/L6938.htm> Acesso em 10/12/2015. 
BRASIL. Constituição Federativa Do Brasil De 1988. Disponível em: $<$ http://www.planalto.gov.br/ccivil_03/Constituicao/Constituicao.htm> Acesso em 10/12/2015.

BRASIL. Decreto nº.99274/90 de 6 de junho de 1990. Regulamenta a Lei nº 6.902, de 27 de Abril de 1981, e a Lei ${ }^{\circ}$ 6.938, de 31 de Agosto de 1981, que dispõem, respectivamente, sobre a criação de Estações Ecológicas e Áreas de Proteção Ambiental e sobre a Política Nacional do Meio Ambiente, e dá outras providências. Diário Oficial da União: Brasília, edição de 7 de junho de 1990. Disponível em: <http://www.planalto.gov.br/ccivil_03/decreto/antigos/d99274.htm> Acesso em 10/12/2015.

BRASIL. MINISTÉRIO DO MEIO AMBIENTE. MMA. Conselho Nacional do Meio Ambiente. CONAMA. $\begin{array}{lllllll}\text { Resolução } & \mathbf{n}^{0} \text {. } & \mathbf{0 0 1}, & 23 & \text { jan., } & 1986 . & \text { Disponível }\end{array}$ <http://www.mma.gov.br/port/conama/res/res86/res0186.html >. Acesso em 10/12/2015.

BRASIL. CONSELHO NACIONAL DE MEIO AMBIENTE. Resolução no 237, de 19 de dezembro de 1997. Estabelece a revisão dos procedimentos e critérios utilizados no licenciamento ambiental, de forma a efetivar a utilização do sistema de licenciamento como instrumento de gestão ambiental, instituído pela Política Nacional do Meio Ambiente. Disponível em: <http://www.mma.gov.br/port/conama/res/res97/res23797.html> Acesso em $10 / 12 / 2015$.

COFFIN, A.W. From roadkill to road ecology: a review of the ecological effects of roads. Journal of Transport Geography. $\quad$ n. $\quad 15, \quad 396-406 . \quad 2007 . \quad$ Disponível <http://www.sciencedirect.com/science/article/pii/S0966692306001177>Acessado em: 12/11/2015.

CLEVENGER, A. P.; CHRUSZCZ, B; GUNSON, K. E. Spatial patterns and factors influencing small vertebrate fauna road-kill aggregations. Biol. Conserv., n.109, p. 15-26. 2003.

CORLATTI, L.; HACKLÄNDER, K.; FREY-ROOS, F. Ability of wildlife overpasses to provide connectivity and prevent genetic isolation. Conservation Biology. n.23 p.548-556. 2009.

FORMAN, R.T.T, ALEXANDER, L.E. Roads and their major ecological effects. Annual Review of Ecology, Evolution and Systematics. n. 29, p. 207-231. 2007. Disponível em: <http://www.annualreviews.org/doi/abs/10.1146/annurev.ecolsys.29.1.207> Acessado em 23/09/2015.

FORMAN, R.T.T, DEBLINGER, R.D. The ecological road-effect zone of a Massachusetts (USA) suburban highway. Conservation Biology. n. 14, p. 36-46. 2000.

FORMAN, R.T.T; SPERLING, D; BISSONETTE, J.A; CLEVENGER, A.P; CUTSHALL, C.D; DALE, V.H; FAHRIG, L; FRANCE, R; GOLDMAN, C.R; HEANUE, K; JONES, J.A; SWANSON, F.J; TURRENTINE, T; WINTER, T.C. Road ecology: science and solutions. Island Press, Washington. 2003.

GONÇALVES, L. O. Avaliações de impacto ambiental de rodovias: as perguntas estão sendo respondidas? Dissertação (mestrado). 211 p. Universidade Federal do Rio Grande do Sul. Porto Alegre, 2012. Disponível em: <http://www.lume.ufrgs.br/handle/10183/72345> Acessado em 07/11/2015.

JONES, J.A; SWANSON, F.J; WEMPLE, B.C; SNYDER, K.U. Effects of roads on hydrology, geomorphology and disturbance patches in stream networks. Conservation Biology. n. 14, p.76-85. 2000.

KOCIOLEK A.V, CLEVENGER A.P, ST-CLAIR C.C, PROPPE D.S Effects of road networks on bird populations. Conserv Biol. n.25, v.2, p.241-249. 2011.

LAUXEN, M.S. A mitigação dos impactos de rodovias sobre a fauna: Um guia de procedimentos para tomada de decisão. Porto Alegre. Monografia apresentada no departamento de zooologia como pré-requisito para a conclusão do curso pós-graduação Lato Sensu, em Diversidade e conservação da fauna. Universidade Federal do Rio Grande do Sul. 176 p. 2012.

LODÉ, T. Effect of a motorway on mortality and isolation of wildlife populations. Ambio, v.29, n.3, p.163-166. 2000. Disponível em: <http://www.bioone.org/doi/abs/10.1579/0044-7447-29.3.163> Acessado em 17/07/2015.

PERZ, S.G; CALDAS, M.M; ARIMA, E; WALKER, R.J. Unofficial road building in the Amazon: socioeconomic and biophysical explanations. Development and Change. n.38, p.529-551. 2007. Disponível em: 〈http://onlinelibrary.wiley.com/doi/10.1111/j.1467-7660.2007.00422.x/abstract > Acessado em 21/08/2015. 


\section{A ECOLOGIA DE ESTRADA SOB A ÓTICA \\ DO LICENCIAMENTO AMBIENTAL}

REIJNEN, R; FOPPEN, R; BRAAK, C.T; THISSEN, J. The effects of car traffic on breeding bird populations in woodland. Reduction of density in relation to the proximity of main roads. Journal of Applied Ecology. n.32 p.187-202. 1995.

SILVA, R.M; BORBA, C.H.O; LEÃO, V.P.C; MINEO, M.F. O impacto das rodovias sobre a fauna de vertebrados silvestres no cerrado mineiro. Enciclopédia biosfera, Centro Científico Conhecer - Goiânia, v.7, n. $12 ; 2011$.

SOARES-FILHO B, ALENCAR .A, NEPSTAD .D, CERQUEIRA. G, DIAZ, M.C.V, RIVERO S, SOLO`RZANO L, VOLL, E. Simulating the response of land-cover changes to road paving and governance along a major Amazon highway: the Santarem-Cuiaba corridor. Global Change Biology n.10 p. 745-764. 2004.

TAYLOR, B. D.; GOLDINGAY, R. L. Wildlife road-kills on three major roads in north-eastern New South Wales. Wildlife Research, Collingwood, v. 31, p. 83-91. 2004. Disponível em: <http://www.publish.csiro.au/paper/WR01110.htm>Acessado em 24/11/2015.

TROMBULAK, S.C; FRISSELL, C.A. Review of ecological effects of roads on terrestrial and aquatic communities. Conservation Biology n. 14, p. 18-30. 2000. 